\title{
Application of Computer-aided Equipment in Voice Science Protection
}

\author{
Qiuyue Fan \\ Musical Department, Heihe College, Heilongjiang, China
}

Keywords: computer-assisted; voice protection

\begin{abstract}
Voice" is just like the life to the singer. How to protect, maintain and make it shine forever is the dream of every singer. Computer-assisted instruction is a new teaching method in many fields at present, which has been applied to the protection of the traditional voice. The application of computer in voice protection is a new means to stimulate vocal learners' interest, but also improve effect and efficiency of voice protection with unique advantages, unmatched by traditional methods. This paper expounds the application of computer in the protection of voice science so as to enable the singer to find the correct way of voice health care.
\end{abstract}

\section{Introduction}

Language is the most important means of human communication, with the development of society, economic prosperity, people's contacts, cultural exchanges and other increasingly frequent, followed by the incidence of voice is also on the rise. The formation of a person's voice takes time, spiritual feeling, honed artistic voice, in the form of before and after the formation of the art of how to protect the voice, and extend its artistic life, it is placed in front of each singer, I analyze from the following several aspects how to protect our artistic voice. A computer aided system is a general term for a system that uses computer aid to accomplish different tasks. Computer aided equipment provides a better condition for the scientific protection of voice. The application of computer technology, such as multimedia, hypertext, artificial intelligence, network communication and knowledge base, has overcome the shortcomings of the traditional protection methods. It can shorten the study time, improve the teaching quality and teaching efficiency, realize the optimized teaching target and promote the scientific protection of voice (Feng, 2006).

\section{Common Voice Problems}

We must first understand the mentioned singing vocal organ, the vocal organs, respiratory organs (lung protection, diaphragm and abdominal muscles) is the dynamic part of the larynx is part of vocal cord vibration, oral, pharyngeal cavity and nasal cavity resonance is part of, in addition to the articulation part, including the lip, tongue and teeth etc. (Yao, 2003). The language of sound is like this, first respiratory movement expiratory breath, breath through the closure of the vocal cords, the vibration of laryngeal vowel the laryngeal acoustic resonance by pharyngeal cavity, oral cavity and nasal cavity, and then by using the lips, teeth, tongue articulation organ, finally with the language and the meaning of singing to voice. Incorrect vocal methods can cause harm to your voice. There are several reasons for the wrong pronunciation:

\subsection{The incorrect breathing method}

Poor respiratory control is an important cause of voice disorders. The wrong way is shallow breath suction or stiff, clavicle type or the thoracic breathing breath is often caused by the lack of flexibility of vocal cord edema, chest, neck stiffness, muscle tension and vascular engorgement, this will affect the articulation organ function best resonance. Correct singing, breathing is controlled, and the chest and belly combine breathing. In the usual training about "sigh" feeling; and bent ninety degrees, down to feel the breath Chuishou upright, singing in the same breath feeling. 


\subsection{Unscientific vocal cord movement}

Disturbance of motion regulation. The vocal movement of the vocal cords in normal people has two states: the heavy tone function and the light tone function. The function is really heavy sound, falsetto vocal cord movement, vocal cord thickening overall vibration, low frequency, large amplitude (Yue, 2012). The function is softly falsetto, falsetto voice vocal cord movement, vocal tension thinning, edge vibration, high frequency, small amplitude. The right vocal cord movement is true with, namely the bass sound more real falsetto less high falsetto voice sound really much less, from low to high, two phonation function gradually conversion, two groups of muscles is a dynamic balance. The correct adjustment of the two functions is necessary for long-term exercise. Excessive use of a function of singing can cause lesions in the vocal cords.

\subsection{The incorrect use of resonance}

The singers in the singing without empathy or sympathy, not only the poor acoustics, lack of vocal fold vibration resonance resonance organ protective impedance, easy movement of excessive laryngeal disease, shouting bad voice sounding type is the truth. With use, should be combined with oral and nasal cavity, thoracic cavity resonance, soprano to nasal mainly with the chest, chest mainly with bass to sing tenor in the nasal cavity, oral and nasal and chest with resonance, the only way to sound unified, strong penetrating power, the resonating organs play the best the role, in addition to the above reasons, the singing training not too much exercise is a treble, the students singing errors on the understanding, think good can show their singing soprano, actually want to sing out good works with many factors, here is not described. The solution of the treble is not too much practice and high pitched voice, but should start with the training of the tenor, grasp the correct and stable singing state, and the high tone will be easily solved. Excessive use of the voice is also easy to make vocal fatigue, some students find good singing feeling crazy practice, no time limit, to find a good singing state when there is no patience to stick with a place for many days, the formation of practice will affect the law that was not a good voice, but also hurt the throat (Huang, 2007).

\section{Voice Protection in Lesions}

When the noise occurs, the lesion is divided into two main cases: one is that the incorrect method of speech can lead to a change of voice; one is that certain conditions on the body may affect the voice. The voice is not correct will make the voice of what disease? Learned singing people all know, a good voice needs two functions, namely weight function and coordination function is light, people often say true voice and falsetto voice. Any single use of a function, or even two functions at the same time, but not in proportion, can cause problems in the throat. If the voice used too much, the chances of vocal polyps; if too many falsetto vocal nodules, long more opportunities. It is very difficult to begin with a sound error. But more days, a little bit, the singer will not feel tired appropriate, don't start with clear timbre hoarse voice. This situation may have stayed a long time. In the end, the vocal cords develop red spots and become red nodules, causing discomfort when singing weak sounds. Sometimes it breaks suddenly, or the pitch is not clear, and a leak occurs. Sometimes the edge of the vocal cords may also produce nodules, which make the vocal cords unable to close. This kind of summary, in the early discovery, must change the singing method, and to stop singing, for long-term rest, otherwise the summary will expand or even become a tumor, and finally to surgery, restitution. In addition to the singing method, sometimes physiological diseases such as swelling of the glandular glands on the throat may also impede the singer's singing, as it prevents inhalation through the nostrils and causes inflammation in his nostrils. Tonsillitis often make singer discomfort and affect the vocal and vocal, in fact, tonsils out and laryngeal glands are an easy job to do, but to specific decisions according to each singer's own situation. Under normal circumstances, the younger the age, the art voice has not yet formed, the earlier the better to remove, the younger postoperative recovery faster and less prone to scarring (Song, 2013). The vocal music level has reached the mature stage of the singer, generally do not consider surgery, such as patients 
with frequent tonsillitis and several times a year and cause other diseases and even endanger the patient's life, so no matter what kind of situation also need decisive operation. Sinusitis is the same. If a singer feels inflamed and has a pressure under his throat, he must stop singing and talking and rest immediately, for this may be bronchitis. Tracheitis can cause vocal cord swelling, throat inflammation in long term should not belittle it, to find out the reasons, smoking, drinking or speak too much, if so, to rest, and talked less, do not drink, do not smoke, in order to make a speedy recovery of tracheitis.

\section{Attention to the Singer's Voice Protection}

The sound is second times sexual gonads developed by the throat in a short period of time grows up and a physiological phenomenon caused by. Children become adults sound period in physiology is referred to as "voic emutation". The changing period can be divided into early puberty, voice and change the sound of the late. Since the voice training should pay attention to the following three points:

\subsection{The selection of songs}

Be appropriate. The range of the song should not be too wide, preferably no more than nine degrees, and the volume and intensity should not be too large. Tune to smooth, avoid "shocked", so as to avoid students shouting, if students feel the high tone can be appropriately reduced. Vocalization, singing by the best teacher guidance, in the appropriate range. In addition, the singing time is not too long, so as to prevent the vocal cords from fatigue and damage.

\subsection{Adjust the breathing method}

The characteristics of the students voice mutation, appropriately adjust the breathing method to meet the needs of the growth of the larynx and vocal cords. Change the shallow breathing when children sing, and train the students to sing in the form of chest and abdominal breathing. Make the students know the correct concept of voice, so after the scientific and systematic training can make students' respiratory and vocal organs are functional exercise, so as to lay a solid basic skills in singing.

\subsection{Practice singing, pay attention to step by step, cannot be eager for success}

Can let the students from the proper and easy to sound natural tone of practicing, usually to sing "hum". Girls in the late voice training, may be appropriate to introduce falsetto, mixed voice training, the development of sound for girls in the future will be very beneficial. In addition, in the middle of the sound, listen to the method of less singing, music theory knowledge, strengthen the music appreciation teaching, so that they can improve the knowledge of music theory, art etc. In the changing period, should take part in physical exercise to improve physical quality, enhance resistance to diseases. In life, we must have the law, pay attention to the overall intake of nutrients, maintain adequate sleep, eat less stimulating food, so as to avoid the stimulation of the respiratory tract.

\section{Computer-aided Equipment in Voice Science Protection}

\subsection{Further develop voice teaching software}

Computer assisted instruction software more as a music worker, learning must be based on their actual situation and the actual situation of the vocal music teaching content closely together. Choose suitable, suitable for voice protection of learning and teaching and teaching materials suitable for teaching software, rather than used to use the software don't rely on ready-made software. Other teachers. Because the teaching plan is based on the teaching objectives, teaching content and student situation, written by the teacher himself. People do not necessarily fit you, teaching software is the same reason (Feng, 1998). Therefore, teachers should pay attention to collecting materials at ordinary times, plus their own teaching ideas. Create your own or work with the 
surrounding colleagues to make teaching software, which is more targeted and more conducive to teaching.

\subsection{Use multimedia pictures to strengthen the digestion of knowledge and take notes}

Students need a certain time span to receive information and master knowledge. Multimedia teaching students who take notes slowly and with limited comprehension often take notes, fail to listen to good lessons, listen to class, and do not take notes. If the teacher only lectures, switches pages, and ignore students digest of knowledge. Will reduce the learning effect. In order to improve the situation, teachers should pay attention to the rhythm of the lecture and the correct speech rate when using the butterfly body. Subtitles should not be too fast to play, but to stay in the form of teaching. Many forms such as classroom teaching, experiment class, period practice, clinical practice, protection teaching and second class are adopted. Simultaneous. We should make full use of modern teaching methods, constantly optimize the teaching process, fully mobilize the initiative of students in learning and improve the effect of voice protection teaching.

\subsection{Use computer software to monitor diet and alcohol and smoke, so as to prevent sound}

Any food that has irritation to the throat should be eaten less, or even eaten before the performance. The spicy, sweet, salty, cold or hot food is to stimulate the voice, to Shensi on such food in daily life. There are delicious fried, diet is easy to cause the throat secretions increase, eating too much starch will make weight increase. Should not eat before singing, singing should not be heavy food to eat, so as not to affect sleep, and make weight gain. The usual diet should eat more nutritious foods, such as fresh vegetables, fruits, and honey, egg can throat food. It is more important to see a doctor than to see a doctor. Generally speaking, a small amount of alcohol occasionally, impact on the voice is small, but the long term and excessive smoking and drinking, have an impact on voice, tobacco contains nicotine, methyl cyanide, nitrite compounds and other harmful substances, on human respiratory tract have a direct stimulating effect, make the singers of the dry throat, itching, phlegm, cough. Because of the stimulation of smoking, vocal cords can present chronic inflammatory reaction, and even make the vocal cord polyp like degeneration, the singer's voice becomes dark, loses luster, even hoarse voice. Drinking is not the direct stimulation of the vocal cords, but vocal posterior arytenoid area, aryepiglottic fold that entrance part of the esophagus, often by the stimulation of alcohol can cause chronic mucosal hyperemia, edema, and then spread to the throat, resulting in the formation of chronic laryngitis, vocal cords, voice changed. The harm of alcohol and tobacco affects not only the vocal organs - the throat, vocal cords, but also the body and mind. The brain due to the toxic effects of these harmful substances caused by fatigue, memory loss, etc. In order to protect the voice and physical and mental health, there is no smoking and drinking hobby singer, it is best not to be contaminated with this habit; there are tobacco loving singers, it is best to use less or give up.

\section{Conclusion}

Computer software is a kind of auxiliary equipment, through software monitor monitoring, timely recording of the knowledge, which can be well protected, so that the singer can achieve better results in voice protection.

\section{Acknowledgement}

This thesis is a research project of philosophy and Social Science in Heilongjiang Province 《Scientific training and health care of voice》, Project number is 16YSC03, Project category is Youth Project

\section{References}

[1] Feng, B.F., 2006. Speech physiology and voice protection. Journal of Central Conservatory of 
Music.

[2] Feng, K. X. 1998. Youth voicemutation feature and voice protection on Journal of Southwestern Normal University (SOCIAL SCIENCE EDITION).

[3] Huang, P., 2007. Computer based voice protection and training on China's off campus education.

[4] Peng Y., 2012. Computer assisted voice protection, 0n voice of the Yellow River.

[5] Song C., 2013. Analysis of the path of using computer voice protection in music lessons on Education Teaching Forum.

[6] Yao, L.H., 2003. On singing mechanism and voice protection on success (Education). 